\title{
SUPPLEMENTARY DOCUMENT FOR DECONVOLUTION OF BASE PAIR LEVEL RNA-SEQ READ COUNTS FOR QUANTIFICATION OF TRANSCRIPT EXPRESSION LEVELS
}

1. Further Discussion of Poisson Mixture Distribution. The twocomponent mixture of Poisson distribution of $X_{k m}^{r}$, for $k \in\left\{i_{1}, \ldots, i_{N_{r}}\right\}$, plays a key role in making the inference of transcript expression levels from $Y_{m}^{r}$ possible. Suppose $X_{k m}^{r}$ follows a simple Poisson distribution with intensity rate $\lambda_{k}$. Then, $Y_{m}^{r}=X_{i_{1} m}^{r}+\cdots+X_{i_{N_{r}} m}^{r}$ follows a simple Poisson distribution with intensity rate $\lambda_{i_{1}}+\cdots+\lambda_{i_{N_{r}}}$. Given $y_{m}^{r}, \lambda_{i_{1}}+\cdots+\lambda_{i_{N_{r}}}$ can be estimated, but the individual intensity rates $\lambda_{i_{1}}, \ldots, \lambda_{i_{N_{r}}}$ usually cannot be uniquely identified or estimated. The two-component mixture of Poisson model for $X_{k m}^{r}$ not only characterizes the reads-generating mechanism at transcript level but also makes the inference of transcript expression levels from the gene level read counts data possible. As will be shown later, given the realization $y_{m}^{r}$ for $m \in E^{r}$ and $r \in \mathcal{R}$, the parameters in model (2.2) can be estimated using the maximum likelihood methods, and the resulting estimates can then be further used to quantify the transcript expression levels. We will also show that it is not necessary to allocate reads to different transcripts anymore, as is commonly done in the literature. In theory, $X_{k m}^{r}$ can be assumed to follow a mixture of Poisson distributions with more than two components, and all methods developed in this article can be extended accordingly.

2. Conditional Distribution of $\boldsymbol{y}_{m}^{r}$. In current real RNA-seq data, however, we found that junction reads are rare relative to non-junction reads. In other words, given a junction read type $r \in \mathcal{J}$, for $m \in E^{r}$, the number of positive $y_{m}^{r}$ 's is small. We postulate that the excessive large number of base pairs with $y_{m}^{r}=0$, for $r \in \mathcal{J}$, is caused by other unknown missing mechanisms, which cannot be properly modeled. Therefore, when estimating the model parameters, it may not be appropriate to use the original distribution of $Y_{m}^{r}$, for $r \in \mathcal{J}$. One approach to solving this difficulty is to consider only positive counts $y_{m}^{r}>0$ and the conditional distribution of $y_{m}^{r}$ given $y_{m}^{r}>0$. One advantage of using the positive counts and their conditional distributions is to avoid the ambiguity caused by difference in fragment length in the definition of $E^{r}$ for $r \in \mathcal{J}$. We define $y_{+}^{r}=\left\{y_{m}^{r}: m \in E_{+}^{r}\right\}$, where $E_{+}^{r}=\left\{m: y_{m}^{r}>0\right\}$. For $r \in \mathcal{J}$, the conditional distribution of $Y_{m}^{r}$ given 
$Y_{m}^{r}>0$ for $m \in E^{r}$ is given as follows.

$$
\begin{aligned}
& p\left(Y_{m}^{r}=y \mid Y_{m}^{r}>0\right)=\left\{\prod_{k=i_{1}}^{i_{N_{r}}}\left[\sum_{j_{k}=1}^{2} p_{k j_{k}} \operatorname{Poi}\left(y ; \lambda_{k j_{k}}\right)\right]\right\} /\left\{1-p\left(Y_{m}^{r}=0\right)\right\} \\
& =\frac{\prod_{k=i_{1}}^{i_{N_{r}}}\left[\sum_{j_{k}=1}^{2} p_{k j_{k}} \operatorname{Poi}\left(y ; \lambda_{k j_{k}}\right)\right]}{1-p\left(Y_{m}^{r}=0\right)} \\
& =\frac{\sum_{j_{1}} \ldots \sum_{j_{N_{r}}}\left[p_{i_{1} j_{i_{1}}} \ldots p_{i_{N_{r}} j_{i_{N}}} \operatorname{Poi}\left(y ; \lambda_{i_{1} j_{i_{1}}}+\ldots+\lambda_{i_{N_{r}} j_{i_{N}}}\right)\right]}{1-\sum_{j_{i_{1}}} \ldots \sum_{j_{i_{N}}} p_{i_{1} j_{i_{1}}} \ldots p_{i_{N_{r}} j_{N_{N_{r}}}} e^{-\lambda_{i_{1} j_{i_{1}}}-\ldots-\lambda_{i_{N}} j_{i_{N_{r}}}}} \text {. }
\end{aligned}
$$

3. Distributions in the Illustrative Example. There are two isoforms in gene DARC, the possible types of reads that can be generated are summarized below.

$$
\left\{\begin{array}{lll}
X_{1 m}^{r_{11}} \sim p_{11} \operatorname{Poi}\left(\lambda_{11}\right)+p_{12} \operatorname{Poi}\left(\lambda_{12}\right) & \text { for } m \in E^{r_{11}} \\
X_{1 m}^{r_{13}} \sim p_{11} \operatorname{Poi}\left(\lambda_{11}\right)+p_{12} \operatorname{Poi}\left(\lambda_{12}\right) & \text { for } m \in E^{r_{13}} \\
X_{1 m}^{r_{33}} \sim p_{11} \operatorname{Poi}\left(\lambda_{11}\right)+p_{12} \operatorname{Poi}\left(\lambda_{12}\right) & \text { for } m \in E^{r_{33}} \\
X_{2 m}^{r_{22}} \sim p_{21} \operatorname{Poi}\left(\lambda_{21}\right)+p_{22} \operatorname{Poi}\left(\lambda_{22}\right) & \text { for } m \in E^{r_{22}} \\
X_{2 m}^{r_{23}} \sim p_{21} \operatorname{Poi}\left(\lambda_{21}\right)+p_{22} \operatorname{Poi}\left(\lambda_{22}\right) & \text { for } m \in E^{r_{23}} \\
X_{2 m}^{r_{33}} \sim p_{21} \operatorname{Poi}\left(\lambda_{21}\right)+p_{22} \operatorname{Poi}\left(\lambda_{22}\right) & \text { for } m \in E^{r_{33}}
\end{array}\right.
$$

Here the intensity rates $\lambda_{11}$ and $\lambda_{21}$ account for the no-coverage or abnormally low coverage at the base pairs of transcripts $T_{1}$ and $T_{2}$, respectively, and the intensity rates $\lambda_{12}$ and $\lambda_{22}$ account for the abundance or the expression levels of transcripts $T_{1}$ and $T_{2}$, respectively.

As discussed previously, $X_{t m}^{r_{u v}}$ 's are not always directly observable. Instead, we only observe the aggregated read counts $Y_{m}^{r_{u v}}$ 's. The relationship between $Y_{m}^{r_{u v}}$ and $X_{t m}^{r_{u v}}$ and the distributions of $Y_{m}^{r_{u v}}$ can be obtained as follows. 


$$
\left\{\begin{array}{l}
Y_{m}^{r_{11}}=X_{1 m}^{r_{11}} \sim \sum_{i=1}^{2} p_{1 i} \operatorname{Poi}\left(\lambda_{1 i}\right) \text { for } m \in E^{r_{11}} ; \\
Y_{m}^{r_{13}}=X_{1 m}^{r_{13}} \sim \sum_{i=1}^{2} p_{1 i} \operatorname{Poi}\left(\lambda_{1 i}\right) \text { for } m \in E^{r_{13}} ; \\
Y_{m}^{r_{22}}=X_{2 m}^{r_{22}} \sim \sum_{i=1}^{2} p_{2 i} \operatorname{Poi}\left(\lambda_{2 i}\right) \text { for } m \in E^{r_{22}} ; \\
Y_{m}^{r_{23}}=X_{2 m}^{r_{23}} \sim \sum_{i=1}^{2} p_{2 i} \operatorname{Poi}\left(\lambda_{2 i}\right) \text { for } m \in E^{r_{23}} ; \\
Y_{m}^{r_{33}}=\sum_{i=1}^{2} X_{i m}^{r_{33}} \sim \sum_{i=1}^{2} \sum_{j=1}^{2} p_{1 i} p_{2 j} \operatorname{Poi}\left(\lambda_{1 i}+\lambda_{2 j}\right), m \in E^{r_{33}}
\end{array}\right.
$$

4. Composite Likelihood Function in the Illustrative Example. For gene $g$ with $k_{g}$ exons $e_{1}, \ldots, e_{k_{g}}$, the collection of candidate transcripts $\mathcal{T}_{g}=\left\{T_{1}, \ldots, T_{N}\right\}$, and the collection of possible read types $\mathcal{R}=\mathcal{N} \cup \mathcal{J}$, given the count data of all possible types $\mathbf{y}=\left\{y_{m}^{r}, m \in E^{r}, r \in \mathcal{R}\right\}$, the likelihood function for the model parameters can be derived as follows. We consider non-junction read types first. For $r \in \mathcal{N}, y^{r}=\left\{y_{m}^{r}: m \in E^{r}\right\}$. Recall that $\mathcal{T}_{g}^{r}=\left\{T_{i_{1}} \ldots T_{i_{N_{r}}}\right\}$ is a sub-collection of $\mathcal{T}_{g}$, which includes the transcripts that can give rise to reads of type $r$. Let $\theta^{r}=\left\{\left(p_{k 1}, p_{k 2}, \lambda_{k 1}, \lambda_{k 2}\right)\right.$ : $\left.k \in\left\{i_{1}, \ldots, i_{N_{r}}\right\}\right\}$, which is the collection of the model parameters associated with the transcripts in $\mathcal{T}_{g}^{r}$. The probability mass function of $y_{m}^{r}$ is given in model (2.2) in the main article. Given $y^{r}$, the likelihood function of $\theta^{r}$ is

$$
\begin{aligned}
& L^{r}\left(\theta^{r} \mid y^{r}\right)=\prod_{m \in E^{r}} \prod_{k=i_{1}}^{i_{N_{r}}}\left[\sum_{j_{k}=1}^{2} p_{k j_{k}} \operatorname{Poi}\left(\lambda_{k j_{k}}\right)\right]=\prod_{m \in E^{r}}\left\{\sum_{j_{i_{1}}}^{2}\right. \\
& \left.\ldots \sum_{j_{i_{N_{r}}}}^{2}\left[\left(p_{i_{1} j_{i_{1}}} \ldots p_{i_{N_{r}} j_{i_{N_{r}}}}\right) \operatorname{Poi}\left(y_{m}^{r} ; \lambda_{i_{1} j_{i_{1}}}+\ldots+\lambda_{i_{N_{r}} j_{i_{N_{r}}}}\right)\right]\right\} .
\end{aligned}
$$

Next, we consider junction read types $r \in \mathcal{J}$. For $r \in \mathcal{J}, y^{r}=\left\{y_{m}^{r}: m \in\right.$ $\left.E^{r}\right\}$. Due to the reasons discussed in Section (2.2), we only use the nonzero counts $y_{m}^{r}>0$ and the conditional distribution of $Y_{m}^{r}$ given $Y_{m}^{r}>0$. We define $\mathcal{T}^{r}$ and $\theta^{r}$ in the same way as those for non-junction types. Given the positive counts $y_{+}^{r}=\left\{y_{m}^{r}: m \in E_{+}^{r}\right\}$, the conditional likelihood function of $\theta^{r}$ is

$$
L_{c}^{r}\left(\theta^{r} \mid y_{+}^{r}\right)=\prod_{m \in E_{+}^{r}, \text { s.t. } y_{m}^{r}>0} p\left(y_{m}^{r} \mid \theta^{r}, y_{m}^{r}>0\right)
$$




$$
=\prod_{m \in E_{+}^{r}, \text { s.t. } y_{m}^{r}>0} \frac{\sum_{j_{i_{1}}}^{2} \ldots \sum_{j_{i_{r}}}^{2} p_{i_{1} j_{i_{1}}} \ldots p_{i_{N_{r}} j_{N_{r}}} \operatorname{Poi}\left(y_{m}^{r} ; \lambda_{i_{1} j_{i_{1}}}+\ldots+\lambda_{i_{N_{r}} j_{i_{N}}}\right)}{1-\sum_{j_{i_{1}}} \ldots \sum_{j_{i_{N}}} p_{i_{1} j_{i_{1}}} \ldots p_{i_{N_{r}} j_{i_{N_{r}}}} e^{-\lambda_{i_{1} j_{i_{1}}}-\ldots-\lambda_{N_{N}} j_{i_{N_{r}}}}},
$$

where $\mathrm{c}$ in $L_{c}^{r}$ indicates conditional likelihood. Assume that there are $\left|\mathcal{T}_{g}\right|=$ $N$ candidate transcripts in $\mathcal{T}_{g}$, which are indexed by $1,2, \ldots, N$. Let $\theta=$ $\left\{\left(p_{i 1}, p_{i 2}, \lambda_{i 1}, \lambda_{i 2}\right): 1 \leq i \leq N\right\}$, which is the collection of all model parameters. We assume read counts of different types are independent with each other. Let $\tilde{\mathbf{y}}=\left\{y^{r}: r \in \mathcal{N}\right\} \cup\left\{y_{+}^{r}: r \in \mathcal{J}\right\}$. Given $\tilde{\mathbf{y}}$, the likelihood function of $\theta$ can be obtained by combining the likelihood functions (4.1) and (4.2) as follows.

$$
L(\theta \mid \tilde{\mathbf{y}})=\prod_{r \in \mathcal{N}} L^{r}\left(\theta^{r} \mid y^{r}\right) \cdot \prod_{r \in \mathcal{J}} L_{c}^{r}\left(\theta^{r} \mid y^{r}, y^{r}>0\right)
$$

We refer to $L(\theta \mid \tilde{\mathbf{y}})$ as the composite likelihood function of $\theta$, since it involves both ordinary likelihood functions and conditional likelihood functions. The maximum composite likelihood estimate (MCLE) of $\theta$ is defined as the solution to the following maximization problem:

$$
\begin{gathered}
\max _{\theta} L(\theta \mid \tilde{\mathbf{y}}), \\
\text { subject to }\left\{\begin{array}{l}
\lambda_{i j}>0, \text { for } 1 \leq i \leq N \text { and } 1 \leq j \leq 2 ; \\
\lambda_{11}=\ldots=\lambda_{N 1} \\
p_{i 1}+p_{i 2}=1 \text { for } 1 \leq i \leq N .
\end{array}\right.
\end{gathered}
$$

The MCLE of $\theta$ is denoted as $\hat{\theta}$. The behavior and properties of general MCLEs have been studied by Reed et al. (Varin, Reid and Firth (2011)). They are consistent, asymptotically normal, and may however trade some efficiency for gain in convenience in modeling and computation. In the next subsection, we use the EM algorithm to compute $\hat{\theta}$. 
5. Likelihood function in illustrative example.

$$
\begin{aligned}
L(\theta \mid \mathbf{y})= & \prod_{r_{u u} \in \mathcal{N}} L^{r_{u u}}\left(\theta^{r_{u u}} \mid y^{r_{u u}}\right) \cdot \prod_{r_{u v} \in \mathcal{J}} L_{c}^{r_{u v}}\left(\theta^{r_{u v}} \mid y^{r_{u v}}\right) \\
= & \prod_{m \in E^{r_{11}}} \sum_{i=1}^{2}\left[p_{1 i} \operatorname{Poi}\left(y_{m}^{r_{11}} ; \lambda_{1 i}\right)\right] \cdot \prod_{m \in E^{r_{22}}} \sum_{i=1}^{2}\left[p_{2 i} \operatorname{Poi}\left(y_{m}^{r_{22}} ; \lambda_{2 i}\right)\right] . \\
& \prod_{m \in E^{r_{33}}} \sum_{i=1}^{2} \sum_{j=1}^{2}\left[p_{1 i} p_{2 j} \operatorname{Poi}\left(y_{m}^{r_{33}} ; \lambda_{1 i}+\lambda_{2 j}\right)\right] . \\
& \prod_{m \in E_{+}^{r_{13}}} \sum_{i=1}^{2}\left[p_{1 i} \cdot \operatorname{Poi}\left(y_{m}^{r_{13}} ; \lambda_{1 i} \mid y_{m}^{r_{13}}>0\right)\right]
\end{aligned}
$$

6. EM Algorithm. Directly optimizing the original composite likelihood function $L(\theta \mid \tilde{\mathbf{y}})$ is difficult and time consuming. Instead we apply the EM algorithm to calculate the MCLE $\hat{\theta}$. In order to develop the EMalgorithm, we introduce a two-step data generating scheme as follows. For type $r \in \mathcal{R}$ and $m \in E^{r}$, recall that $Y_{m}^{r}$ follows a $2^{N_{r}}$-component mixture of Poisson distribution, and we index the components by $i_{1} j_{i_{1}} \ldots i_{N_{r}} j_{i_{N_{r}}}$, for $j_{i_{k}} \in\{1,2\}$ and $k \in\left\{1, \ldots, N_{r}\right\}$. We define membership indicator variables $Z_{m,\left(i_{1} j_{i_{1}} \ldots i_{N_{r}} j_{i_{r}}\right)}^{r}$ such that $Z_{m,\left(i_{1} j_{i_{1}} \ldots i_{N_{r}} j_{N_{N_{r}}}\right)}^{r}=1$ if $Y_{m}^{r}$ is from the component of convolution distribution with intensity $\left(\lambda_{i_{1} j_{i_{1}}}+\ldots+\lambda_{i_{N_{r}} j_{N_{r}}}\right)$; and $Z_{m,\left(i_{1} j_{i_{1}} \ldots i_{N_{r}} j_{i_{r}}\right)}^{r}=0$, otherwise. Let $z^{r}=\left\{z_{m,\left(i_{1} j_{i_{1}} \ldots i_{N_{r}} j_{i_{N}}\right)}^{r}, m \in E^{r}\right\}$, for $r \in \mathcal{N}$ and $z_{+}^{r}=\left\{z_{m,\left(i_{1} j_{i_{1}} \ldots i_{N_{r}} j_{N_{r}}\right)}^{r}, m \in E_{+}^{r}\right\}$ for $r \in \mathcal{J}$. Let $\tilde{\mathbf{z}}=\left\{z^{r}: r \in\right.$ $\mathcal{N}\} \cup\left\{z_{+}^{r}: r \in \mathcal{J}\right\}$, which is the membership indicator of $\tilde{\mathbf{y}}$. With both $\tilde{\mathbf{y}}$ and $\tilde{\mathbf{z}}$, the complete composite log-likelihood for $\theta$ can be written as

$$
\begin{gathered}
l(\theta \mid \tilde{\mathbf{y}}, \tilde{\mathbf{z}})=\log \left(L\left(\theta^{r} \mid \mathbf{y}, \mathbf{z}\right)\right) \\
=\sum_{r \in \mathcal{N}} l^{r}\left(\theta^{r} \mid y^{r}, z^{r}\right)+\sum_{r \in \mathcal{J}} l_{c}^{r}\left(\theta^{r} \mid y^{r}, z^{r}, y^{r}>0\right),
\end{gathered}
$$


where for non-junction type $r \in \mathcal{N}$, the complete log-likelihood is

$$
\begin{aligned}
& l^{r}\left(\theta^{r} \mid y^{r}, z^{r}\right)=p\left(y^{r}, z^{r} \mid \theta^{r}\right) \\
& =\sum_{m \in E_{r}}\left\{\sum _ { j _ { i _ { 1 } } } \ldots \sum _ { j _ { i _ { N _ { r } } } } ( z _ { m , i _ { 1 } j _ { i _ { 1 } } \ldots i _ { N _ { r } } j _ { i _ { N _ { r } } } } ^ { r } ) \cdot \left[\operatorname { l o g } \left(p_{i_{1} j_{i_{1}}}\right.\right.\right. \\
& \left.\left.\left.\ldots p_{i_{N_{r}} j_{N_{N_{r}}}}\right)+\log \left(\operatorname{Poi}\left(\lambda_{i_{1} j_{i_{1}}}+\ldots+\lambda_{i_{N_{r}} j_{N_{N_{r}}}}\right)\right)\right]\right\}
\end{aligned}
$$

and for junction read type $r \in \mathcal{J}$, the complete conditional log-likelihood is

$$
\begin{aligned}
& l_{c}^{r}\left(\theta^{r} \mid y^{r}, z_{+}^{r}, y^{r}>0\right)=p\left(y^{r}, z_{+}^{r} \mid \theta^{r}, y^{r}>0\right)=\sum_{m \in E_{+}^{r}} \\
& \left\{\sum _ { j _ { i _ { 1 } } } \ldots \sum _ { j _ { i _ { N } } } ( z _ { m , i _ { 1 } , j _ { i _ { 1 } } \ldots i _ { N _ { r } } j _ { i _ { N _ { r } } } } ^ { r } ) \cdot \left[\log \left(p_{i_{1} j_{i_{1}}} \ldots p_{i_{N_{r}} j_{i_{N_{r}}}}\right)+\right.\right. \\
& \left.\log \left(\operatorname{Poi}\left(\lambda_{i_{1} j_{i_{1}}}+\ldots+\lambda_{i_{N_{r}} j_{i_{N_{r}}}}\right)\right)\right]-\sum_{j_{i_{1}}} \ldots \sum_{j_{i_{N_{r}}}}\left(z_{m, i_{1} j_{i_{1}} \ldots i_{N_{r}} j_{i_{N_{r}}}}^{r}\right) . \\
& \log \left(1-p_{i_{1} j_{i_{1}}} \ldots p_{i_{N_{r}} j_{N_{r}}} \cdot e^{\left.-\lambda_{i_{1} j_{i_{1}}} \ldots-\lambda_{i_{N_{r}} j_{N_{N_{r}}}}\right)}\right\} .
\end{aligned}
$$

Suppose the current parameter estimate is $\hat{\theta}^{\text {cur }}=\left(\hat{\lambda}^{\text {cur }}, \hat{p}^{\text {cur }}\right)^{\prime}$. The E-step is to calculate the expected complete log-likelihood function $Q\left(\theta \mid \hat{\theta}^{\text {cur }}, \tilde{\mathbf{y}}\right)=$ $E_{\tilde{\mathbf{z}}}\left[\log \left(l\left(\theta \mid \hat{\theta}^{c u r}, \tilde{\mathbf{y}}, \tilde{\mathbf{z}}\right)\right)\right]$, where the expectation is over the conditional distribution of $\tilde{\mathbf{z}}$ given $\hat{\lambda}^{\text {cur }}, \hat{p}^{\text {cur }}$, and $\tilde{\mathbf{y}}$. Notice that $Q\left(\theta \mid \hat{\theta}^{\text {cur }}, \tilde{\mathbf{y}}\right)$ can also be written as $\left.E\left[\sum_{r \in \mathcal{N}} l^{r}\left(\theta^{r} \mid y^{r}, \hat{\theta}^{c u r}, z^{r}\right)+\sum_{r \in \mathcal{J}} l_{c}^{r}\left(\theta^{r} \mid y^{r}, \hat{\theta}^{c u r}, z_{+}^{r}, y^{r}>0\right)\right)\right]$. The function $Q$ consists of two parts, one of which involves the non-junction types, and the other involves the junction types. The expectation of $z^{r}$ for $r \in \mathcal{N}$ and $z_{+}^{r}$ for $r \in \mathcal{J}$ can be calculated separately. For $r \in \mathcal{N}$, it is to compute

$$
\begin{aligned}
& E\left(z_{m, i_{1} k_{i_{1}} \ldots i_{N_{r}} k_{i_{N}}}^{r} \mid \hat{\lambda}^{\text {cur }}, \hat{p}^{\text {cur }}, y_{m}^{r}\right) \\
& =\frac{\hat{p}_{i_{1} k_{i_{1}}}^{c u r} \ldots \hat{p}_{i_{N_{r}} k_{i_{r}}}^{\text {cur }} \operatorname{Poi}\left(\hat{\lambda}_{i_{1} k_{i_{1}}}^{c u r}+\ldots+\hat{\lambda}_{i_{N_{r}} k_{i_{N_{r}}}^{c u r}}^{c u}\right)}{\sum_{j_{i_{1}}} \ldots \sum_{j_{i_{N_{r}}}}\left[\hat{p}_{i_{1} j_{i_{1}}}^{c u r} \ldots \hat{p}_{i_{N_{r}} j_{i_{N_{r}}}}^{\text {cur }} \operatorname{Poi}\left(\hat{\lambda}_{i_{1} j_{i_{1}}}^{c u r}+\ldots+\hat{\lambda}_{i_{N_{r}} j_{i_{N_{r}}}}^{c u r}\right)\right]},
\end{aligned}
$$


and for $r \in \mathcal{J}$, it is to compute

$$
\begin{aligned}
& E\left(z_{m, i_{1} k_{i_{1}} \ldots i_{N_{r}} k_{i_{N}}}^{r} \mid \hat{\lambda}^{\text {cur }}, \hat{p}^{\text {cur }}, y_{m}^{r}, y_{m}^{r}>0\right) \\
& =\frac{\hat{p}_{i_{1} k_{i_{1}}}^{c u r} \ldots \hat{p}_{i_{N_{r}} k_{i_{N_{r}}}}^{c u r} \operatorname{Poi}\left(\hat{\lambda}_{i_{1} k_{i_{1}}}^{c u r}+\ldots+\hat{\lambda}_{i_{N_{r}} k_{i_{N_{r}}}^{c u r}}^{c u}\right)}{\sum_{j_{i_{1}}} \ldots \sum_{j_{i_{N_{r}}}}\left[\hat{p}_{i_{1} j_{i_{1}}}^{c u r} \ldots \hat{p}_{i_{N_{r}} j_{i_{N_{r}}}}^{\text {cur }} \operatorname{Poi}\left(\hat{\lambda}_{i_{1} j_{i_{1}}}^{c u r}+\ldots+\hat{\lambda}_{i_{N_{r}} j_{N_{N}}}^{c u r}\right)\right]} .
\end{aligned}
$$

The M-step is to maximize $Q$ with respect to $\lambda$ and $p$, and the resulting maximizers can be used to update $\hat{\theta}^{\text {cur }}=\left(\hat{\lambda}^{\text {cur }}, \hat{p}^{\text {cur }}\right)^{\prime}$. We use a block coordinate descent algorithm to optimize $Q$. First, we fix the value of $p$ at $\hat{p}^{\text {cur }}$ and maximize $Q$ with respect to $\lambda$. The resulting maximizer is $\left(\hat{\lambda}_{11}, \hat{\lambda}_{12}, \ldots, \hat{\lambda}_{N 2}\right)=\arg \max _{\lambda_{11}, \lambda_{12} \ldots, \lambda_{N 2}} Q$, and the current estimate of $\hat{\lambda}^{\text {cur }}$ is updated to be $\left(\hat{\lambda}_{11}, \hat{\lambda}_{12}, \ldots, \hat{\lambda}_{N 2}\right)$. Second, we fix the the value of $\lambda$ at $\hat{\lambda}^{\text {cur }}$, and optimize $Q$ with respect to $p_{t 2}$ for $1 \leq t \leq N$. Let $\hat{p}_{t 2}=\arg \max _{p_{t 2}} Q$ and then $\hat{p}_{t 1}=1-\hat{p}_{t 2}$ for $1 \leq t \leq N$. Then current estimate $\hat{p}^{\text {cur }}$ is updated to be $\left(\hat{p}_{12}, \ldots, \hat{p}_{N 2}\right)$.

The EM algorithm iterates between the E-step and M-step until some convergence criterion is satisfied. It is worth pointing out that as the number of candidate transcripts increases, the computational complexity also increases. The EM algorithm for the CPM-Seq model suffers from the curse of dimensionality and the problem of local optima. To deal with the first problem, more sophisticated optimization algorithms or parallel computing techniques could be implemented. To deal with the second problem, we adopt the strategy of using multiple initializations. We repeat the EM algorithm with different initial values of the parameters and choose the estimates that achieve the largest likelihood value.

7. Quantification. Suppose the MCLEs of the model parameters for transcript $T_{t}$ are calculated to be $\hat{\lambda}_{t 1}, \hat{\lambda}_{t 2}, \hat{p}_{t 1}$, and $\hat{p}_{t 2}$. Following the quantification procedure proposed by Wu et al. (Wu, Qin and Zhu (2012)), the expression level of transcript $T_{t}$ is quantified to be $g_{t}^{s}=\left(s \hat{\lambda}_{t 1} \hat{p}_{t 1}+\right.$ $\left.\hat{\lambda}_{t 2} \hat{p}_{t 2}\right) /\left(s \hat{p}_{t 1}+\hat{p}_{t 2}\right)$, where $s$ is a pre-specified number between 0 and 1 . When $s$ is close to $0, g_{t}^{s}$ is dominated by the component that mainly represents transcript's abundance, and $g_{t}^{s}$ is sensitive but suffers from high variability. When $s$ is close to $1, g_{t}^{s}$ is dominated by the component that mainly represents background noise or zero counts, and $g_{t}^{s}$ is stable but insensitive to the transcript's expression level. A proper value of $s$ can avoid the two extremes and lead to a sensitive as well as robust quantification result of 
the transcript. As proposed in Wu et al. (Wu, Qin and Zhu (2012)), using single isoform genes, a bootstrap procedure can be used to find the proper value of $s$. In practice, we found that a $s$ value between 0.2 and 0.3 will in general be a good choice.

8. Illustrative Example Continued. For gene DARC, the annotated two transcripts are able to generate five types of reads. The annotation and the base pair level counts of three non-zero types of reads are plotted in the following two figures.

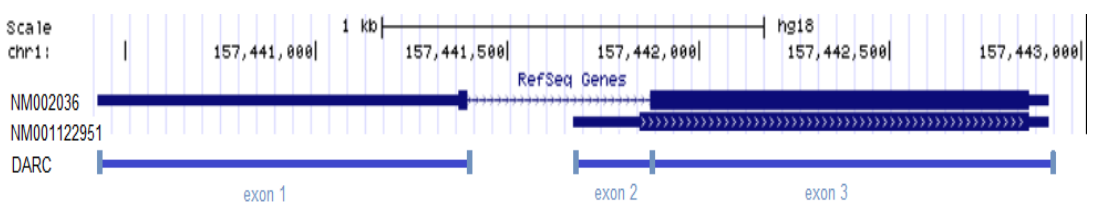

Fig 1: Annotation of DARC in Illustrative Example
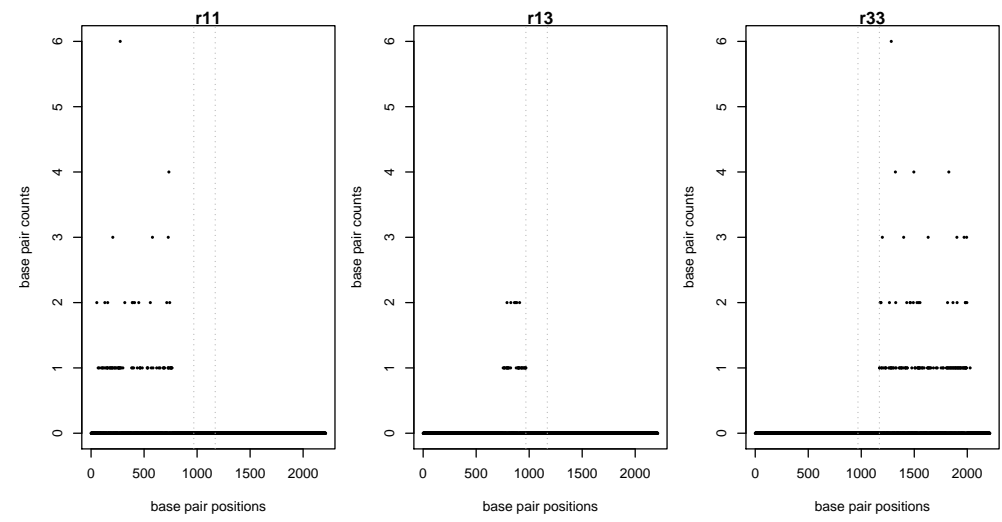

Fig 2: Base level counts of each type of reads in DARC in Illustrative Example

9. Quantification Results for MCF7 in Example 4. The Spearman rank correlation coefficients between the gold standard and the three quantification methods based on the HME cell line are calculated and reported in Table 4. 
TABLE 1

Quantification results of $q R T-P C R, C P M-S e q$, and Cufflinks for eight transcripts in

Example 4

\begin{tabular}{l|cccc}
\hline & \multicolumn{4}{|c}{ MCF7 } \\
\hline transcript ID & qRT-PCR & CPM-Seq & Cufflinks & RSEM \\
\hline uc002cvs.1 & 595.4 & 1.7365 & 2.7964 & 3.66 \\
uc002cvt.2 & 302.3 & 1.5082 & 51.6319 & 66.77 \\
uc002qlp.1 & 381.3 & 2.0921 & 17.1806 & 15.41 \\
uc002qlq.1 & 755.9 & 2.7526 & 44.7393 & 50.07 \\
uc002xmo.1 & 189.6 & 1.6722 & 7.7976 & 11.75 \\
uc002xmn.1 & 530.0 & 3.8728 & 18.7706 & 23.07 \\
uc003ngr.1 & 317.8 & 3.9782 & 125.3420 & 96.59 \\
uc003ngs.1 & 19207.9 & 4.4089 & 45.9333 & 53.63 \\
\hline
\end{tabular}

10. Figure for Example 1. The scatter plots of the CPM-Seq and Cufflinks measurements against those of the gold standard are given in Figure 3. Clearly, the high Pearson correlation is driven by the outlier. However, the Spearman rank correlation is less affected by the outlier. Once we remove the outlier, the Spearman rank correlation coefficient reduced from 0.871 to 0.809 for CPM-Seq and the Spearman rank correlation coefficient reduced from 0.275 to 0.108 for Cufflinks. 
(a)

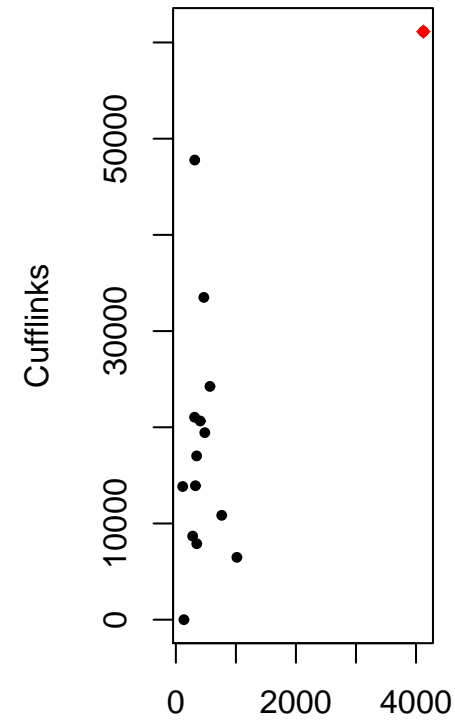

Gold Standard (b)

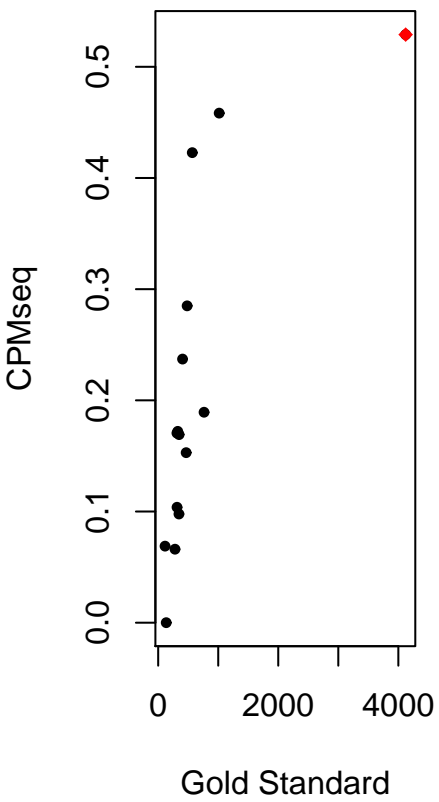

Fig 3: Expression level quantification of 15 transcripts using simulated paired-end data. Plot (a) and (b) show Cufflinks measurements and CPMSeq measurements against the gold standard, respectively 


\section{References.}

VArin, C., Reid, N. and Firth, D. (2011). An Overview of Composite Likelihood Methods. Statistica Sinica 21 5-42.

Wu, H., Qin, Z. S. and Zhu, Y. (2012). PM-Seq: Using Finite Poisson Mixture Models for RNA-Seq Data Analysis and Transcript Expression Level Quantification. Statistics in Biosciences 5 71-87. 